\title{
Design of a Rotary Sprout Vertical Cultivation Device
}

\author{
Yi GUO ${ }^{1, a}$, Jiwu MA ${ }^{2, b}$ \\ ${ }^{1}$ Department of Horticulture, Beijing Vocational College of Agriculture, Beijing, 102442, China \\ ${ }^{2}$ Fengtai District Agriculture and Forestry Mechanical Technology Promotion Station, Beijing, \\ 100070, China
}

aguoyibvca@163.com, bhyie1234@sina.com

Keywords: rotary; sprout; vertical cultivation

\begin{abstract}
Sprouts need substantive water in the growth process, which will cause a high manual irrigation cost. Besides, manual irrigation is usually uneven. To solve this problem, a rotary sprout vertical cultivation device is designed to realize automatic irrigation and guarantee evenness of irrigation during growth of the sprouts. Test results show that this device can save more than 35\% of irrigation water, raise sprout yield by more than $8 \%$ and reduce the labor cost by more than $30 \%$ against the manual irrigation. It features a reasonable structure, reliable working performance, and a beautiful outline. It can meet the demand from agricultural development in cities and thus is easy to be promoted in sprout production and display.
\end{abstract}

\section{Introduction}

Vertical cultivation has combined the cultivation techniques with engineering equipment through facilities engineering, to realize the vertical, inclined and multilayer vertical planting of vegetables. Sunlight greenhouse, a vital part of agricultural facilities, functions in raising the yield of vegetables and extending the supply period of vegetables. Currently the sunlight greenhouse has a primal problem of low land utilization rate. By contrast, the vertical cultivation method can evidently enhance the land utilization rate and raise the yield [1,2]. Guo Yi [3] and et al. developed a layer-rack aeroponic cultivation device that features reliable application and handy operation and is applicable to cultivation of sprouts of different scales. Wang Chunling [4] and et al. developed an H-type vertical cultivation rack with merits of simple construction, easy management and long service life. Yan Zhiming [5] and et al. developed the A-type and ridge-type vegetable vertical cultivation devices that could take full advantage of space and evidently raise the per unit yield. Guan Chunsong [6] and et al. developed a smooth displacement cultivation rack, which can realize translational movement from right to left of multiple racks at the same time and feature simple operation, handiness, thus being able to meet the specific demand from strawberry cultivation. Chen $\mathrm{Na}$ [7] and et al. designed and developed a vertical cultivation rack automatic feed control system that combines hardware and software to realize automatic feedwater and supplemental lighting control. Guo Yi [8] and et al. developed a sprout aeroponic cultivation complete device that utilizes the vertical cultivation technology, aeroponic cultivation technique, ozone sterilization technology, hydromagnetic technology and automatic control engineering and features safety, reliability, high space utilization ratio and autocontrol.

Along with social development and growth in the living standard, people demand vegetables not for the basic living purpose, but the healthy ecology [9]. In this case, people pay more attention to the quality, appearance and security of vegetables and pose more and more demand for health protection properties and functionalities of vegetables [10,11]. Sprouts are "living vegetables" having the antioxidation function. They have unique taste, fresh and tender mouthfeel, rich nutrition and multiple amino acids, mineral substances and vitamins [12,13]. They also function in stomach purging, blood fat reduction and blood pressure lowering, thus presenting a broad market prospect $[14,15]$.

Sprouts require substantive water in their growth process, at least 3 times of irrigation every day. Obviously, they will produce a high labor cost. Besides the manual irrigation features poor evenness. 
A sprout water-saving irrigation device has been designed here to realize automatic irrigation in the sprout growth process and produce even irrigation effect. In addition, it has a beautiful outline and is able to meet the demand from agricultural in cities. As a result, it is easy to be promoted in sprout production and display.

\section{General Planning and Working Process}

The device is composed of the base, irrigation system, rotary rack system, driving system, and control system (Fig. 1). Inside the base is installed with the water tank, water pump and filter of the driving system and the irrigation system and the upside is designed for supporting the rotary rack system. The irrigation system is designed for sprinkling irrigation on sprouts on the rotary rack system. The rotary rack system is used for cultivating the sprouts. In the working process, the system can rotate so that the irrigation system can irrigate all sprouts on the rack. The driving system is to drive the main shaft, which will rotate to drive the entire rotary rack system. The control system is used to set the irrigation period to control the work of the irrigation system and the rotary rack system.

First, the control system is used to set the automatic irrigation period, which can be used for a long time or is subject to change as required. When the control system has reached the irrigation starting time as set, it will start the driving system and the irrigation system, with the former driving the rotary rack and the latter spraying on sprouts on the rotary rack. The rotary of the main shaft enables the complete irrigation of all sprouts. When the irrigation end time is reached as set in the control system, the irrigation system will stop spraying water, but the driving system continues to work until the main shaft rotates for $45^{\circ}$. After irrigation every time, the position of the rotating tray will be changed by $45^{\circ}$, so that sprouts on all positions can get equal illumination.

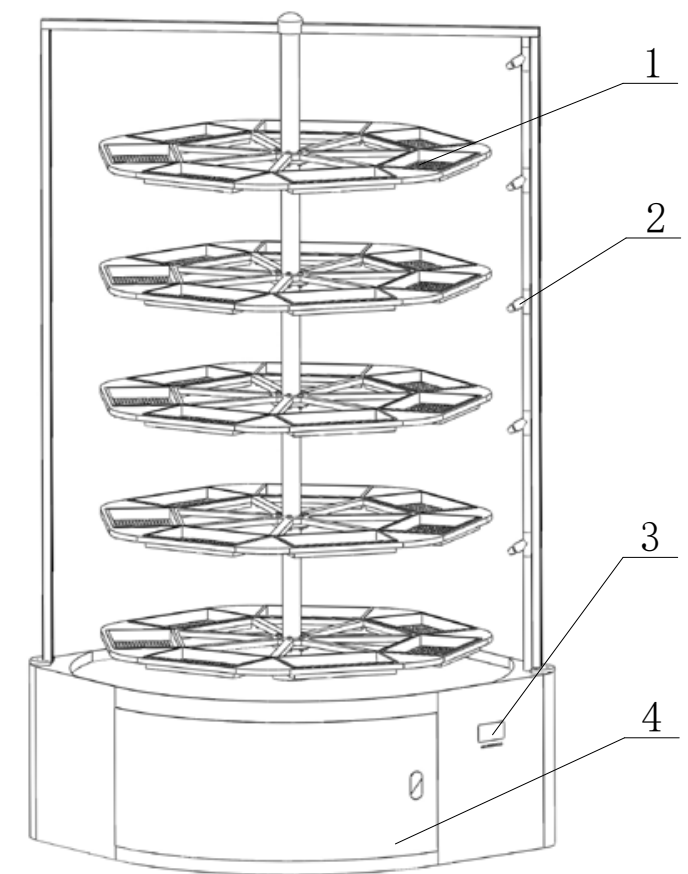

1-rotary rack system 2-irrigation system 3-control system 4- base (including driving system)

Fig. 1 Rotary sprout vertical cultivation device

\section{Structural Design}

Design of the Base. The base is a stainless steel cabinet structure equipped with strengthening rib to raise its weight capacity. To make the device more beautiful and ensure the unanimous effect of the entire device, the base section is designed to be sub-circular. The upper edge of the base is designed with bulges to form a water catcher. The water catcher can store the excessive irrigation water dripped from the seedling tray, which will be returned to the water tank through the return port. In 
the return port, a sponge is designed for filtration to prevent impurities from entering the water tank. On the side of the base there is a gate to facilitate maintenance of the driving system and water tank, water pump and filter of the irrigation system installed inside. On one side of the base there is a rectangular orifice for installing the display of the control system. The structure of the base is shown in Fig. 2.

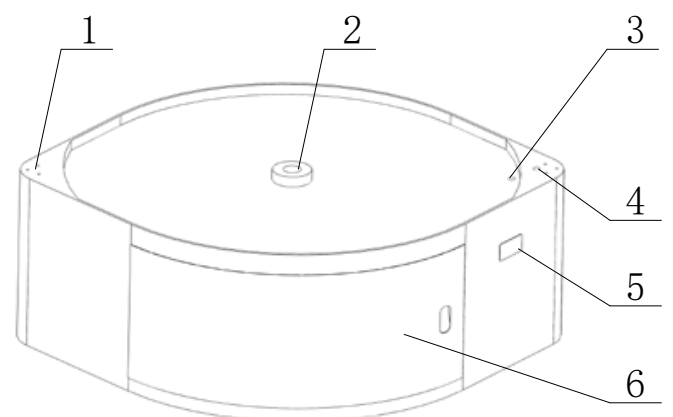

1-sprinkling irrigation support mounting hole 2-main shaft mounting hole 3-backwater hole 4-sprinkling irrigation tube mounting hole 5-display mounting hole 6- base gate

Fig. 2 Base

Design of the Irrigation System. The irrigation system is composed of the water tank, water pump, filter, sprinkling irrigation tube, spray head and support (Fig. 3). The water tank is installed inside the base for storing irrigation water. One tray of sprouts needs $1 \mathrm{~L}$ water on average every day. Thus the water storage capacity of the water tank shall be at least 30L, which will meet the water consumption need from 28 trays of sprouts in the cultivation device. The water pump is used to pressurize water and realize spraying and has adopted the $100 \mathrm{~W}$ high-pressure self-priming pump. The filter is used to screen out impurities in water and adopts the segmented filter. The sprinkling irrigation tube adopts PVC-U water supply pipe and is installed with the micro-fog spray head, with each rotating tray having one spray head, and totally 5 spray heads. The support adopts welded stainless steel square tubes and the sprinkling irrigation tubes are installed on the upright post on one side. Two terminals of the support are installed with flanges on the base. The middle of the support has bearing housing for limiting displacement of the main shaft of the rotary rack system so that the main shaft will be kept in an upright state.

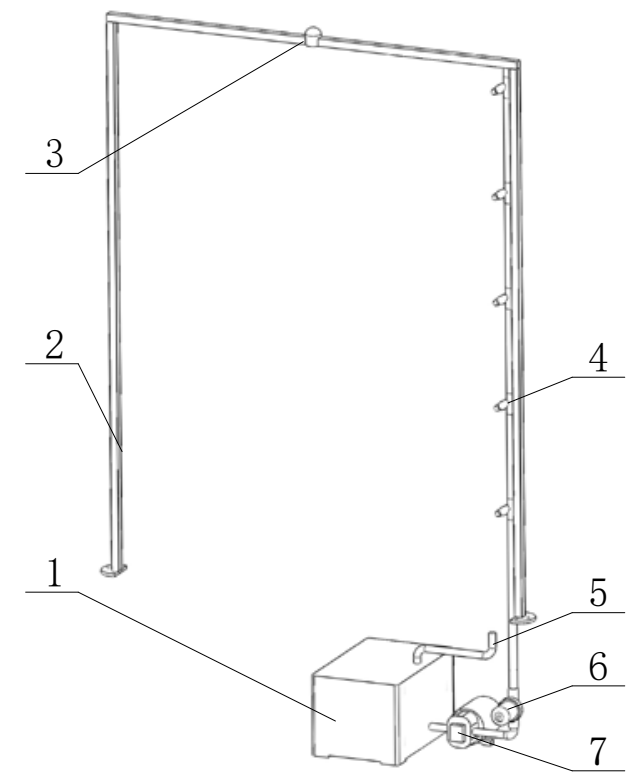

1-water tank 2-support 3-bearing housing 4-sprinkling irrigation tube 5-return pipe 6-filter 7-water pump

Fig. 3 Irrigation system

Design of the Rotary Rack System. The rotary rack system is composed of the main shaft, rotating tray and the seedling tray (Fig. 4). The main shaft is connected with the rotating tray with bolts and fastened with the support bearings and the base bearings. The main shaft is driven by the driving system and then drives the rotating tray. On the rotary rack there are 5 rotating trays, each 
having 7 seedling trays, so the entire rotary rack has 35 seedling trays. The seedling tray adopts the sprout planting tray, with dimension $200 * 300 * 35 \mathrm{~mm}\left(L^{*} W^{*} H\right)$. The diameter of the rotating tray is $1060 \mathrm{~mm}$ to ensure even irrigation.

Design of the Driving System. The driving system is composed of the motor and reducer. When the device works, the motor rotates and is slowed down bit the reducer to drive the main shaft. To ensure even irrigation, the rotary angular velocity of the rotary rack is designed to be 0.1 radian/second. Based on the equation $n=\omega / 2 \pi * 60$, the rotary speed of the main shaft is 0.96 evolution per minute.

Design of the Control System. The control system is composed of the main control chip, display and the electric relay. The main control chip adopts the 51 SCM, which controls the open and close time with the program. Users may set the time with buttons to meet irrigation demands of different kinds of sprouts.

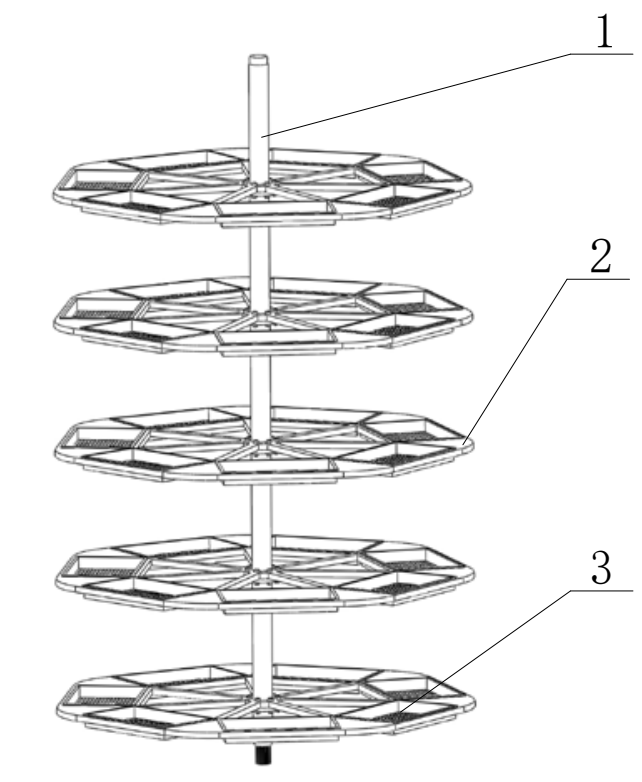

1-main shaft 2-rotating tray 3-seedling tray

Fig. 4 Rotary rack system

\section{Conclusion}

The rotary sprout vertical cultivation device designed here was tested in Beijing Green Valley Sprouts Co, Ltd. Test results show that this device can save more than $35 \%$ of irrigation water, reduce the labor cost by more than $30 \%$ owing to the use of automatic irrigation against the manual irrigation. Besides, the sprinkling irrigation can increase irrigation evenness and thus improve yield by more than $8 \%$ against the manual irrigation. It features a reasonable structure, reliable working performance, and a beautiful outline. It can meet the demand from agricultural development in cities and thus is easy to be promoted in sprout production and display.

\section{Acknowledgements}

This paper is funded by "Water-saving Irrigation Technology in the Production of Sprouts" project (Project No. 20150203-13) under Beijing Municipal Commission of Rural Affairs.

\section{References}

[1] Zhang Xiaowen, Yao Tao, Chen Lizhen, Bu Yunlong, Zhou Zengchan, Li Shushan. Design of Three-dimensional Culture Leaf Device Control System[J]. Agricultural Engineering. 2017(01):38-41.

[2] Xu Xiangpeng, Li Kai, Yang Yanli, Chu QI, Peng Yuping, Feng Jie, Gu song. Development of 
Stereoscopic Cultivation Production Line System [J]. Modern Agricultural Equipment. 2014, (06): 46-49.

[3] Guo Yi, Chen Lanfen, Zhang Guiqin. Design of a Sprout Layer-rack Aeroponic Cultivation Device[J]. Advances in Engineering Research. 2016(104):395-400.

[4] Wang Chungling, Song Weitang, Zhao Shumei, Qu Mingshan. Effect of different combinations of two H-type cultivation frames on light and strawberry growth and yield[J]. Transactions of the Chinese Society of Agricultural Engineering (Transactions of the CSAE). 2017, 33(2): 234-239.

[5] Yan Zhiming, Shi Honglin. Development and Application of Two Types of Balcony Vegetable Hydroponic Contraptions[J]. Southern Horticulture. 2016, 27(1): 35-39.

[6] Guan Chunsong, Hu Hui, Yang Yating, Gao Qingsheng, Zhao Jinyuan. Design and Experiment on Strawberry Moving Cultivation Frame[J]. Journal of Agricultural Mechanization Research. 2017(05):167-170+175.

[7] Chen Na, Chen Liping, Li Bin, Guo Wenzhong, Xue Xuzhang. The Design and Implementation for Dimensional Cultivation Automatic Control System of Balcony Agriculture. Journal of Agricultural Mechanization Research. 2014(01): 127-131.

[8] Guo Yi, Chen Lanfen, Suo Lang Jin Zong, Yang Zhen. Design of Sprout Aeroponics Equipment [J]. Advances in Engineering Research. 2016(88):1783-1789.

[9] Li Zongzhe, Li Deyuan, Su Dan, Ge Zhengwei. The Production Status and Development Strategies of Sprouts [J]. Food Research And Development. 2015(23): 193-196.

[10] Zhao Xia, Wang Feng, Ye Lin. Different Treatment on the Influence of Growth and quality of Medicago sativa Sprouts. Jiangsu Agricultural Sciences. 2015(08):139-141.

[11] Long Dongling, Zhu Qiujin, Zhou Wenmei, Wei Kezhou. Different Treatment on the Influence of Growth and Yield of Perilla Frulescens Sprouts [J]. Journal of Guizhou University (Natural Sciences). 2016(03):35-38.

[12] Guo Yi, Zhang Guiqin. Development of A Design System for the Sprout Vertical Cultivation Device [J]. Advances in Engineering Research. 2015(26):206-211.

[13] Zhang Jie, Ruan XianLe. Study on Biological Characters and Nutritional Changes of Amaranth Sprout[J]. Northern Horticulture. 2013(3): 9-12.

[14] Wang YuanJun. Comparative Study on the Antioxidant Activity of Five Kinds of Sprouts [J]. Soybean Science. 2015(02):260-263.

[15] Tan Zizhen, Li Qingzhou. Disquisition on Application Feasibility of Seedling Vegetable Planting on High Seas Vessels [J]. Journal of Anhui Agri. Sci. 2015,43(2): 391-392. 